\title{
Time Evolution for Infinitely Many Hard Spheres
}

\author{
Roger Alexander $\star$
}

University of Colorado, Boulder, CO 80309, USA

\begin{abstract}
We construct the time evolution for infinitely many particles in $\mathbb{R}^{v}$ interacting by the hard-sphere potential

$$
\Phi(x)=\left\{\begin{array}{cc}
+\infty & |x|<a \\
0 & |x| \geqq a
\end{array}\right.
$$

Because there are abundant examples of hard-sphere configurations with more than one solution to the Newtonian equations of motion, we introduce the concept of a regular solution, in which the growth of velocities and crowding of particles at infinity are limited. We prove that (1) regular solutions exist with probability one in every equilibrium state, and (2) any configuration of the infinite system is the initial point of at most one regular solution. Equilibrium states are invariant under the time-evolution.
\end{abstract}

\section{Introduction}

0.1 . Imagine infinitely many billiard balls of mass $m$ and diameter $a$ at positions $q_{i} \in \mathbb{R}^{v}, i=1,2, \ldots$ (where $\left|q_{i}-q_{j}\right| \geqq a$ if $i \neq j$ ), with corresponding momenta $p_{i} \in \mathbb{R}^{v}$, $i=1,2, \ldots$. The problem is to solve the Newtonian equations of motion when these particles interact by the hard-sphere potential

$$
\Phi(x)=\left\{\begin{array}{cc}
+\infty & |x|<a \\
0 & |x| \geqq a .
\end{array}\right.
$$

The equations of motion take, roughly speaking, the form

$$
\begin{aligned}
& \dot{q}_{i}=p_{i} / m \\
& \dot{p}_{i}=0+\text { elastic reflection at collisions } .
\end{aligned}
$$

Rigorous work on time-evolution of classical systems of infinitely many particles was pioneered by Lanford [4-5]. Lanford's 1974 Battelle lectures [6] contain

* Part of this work forms part of the author's doctoral dissertation written at the University of California, Berkeley, under the direction of O. E. Lanford III 
his construction of time-evolution for particles in $v$-dimensions with long range superstable interactions, as well as a summary of other approaches. Hard core systems have been studied by Ya. Sinai, who constructed dynamics for infinitely many particles in one dimension [10], and in v-dimensions at low density [11], when the interaction is finite-range and singular at the hard core radius.

There is no hope of solving the Equations (0.1.2) for all initial configurations. With the naked hard-sphere potential $(0.1 .1)$ the positions and momenta of outgoing particles after grazing or triple collisions do not depend smoothly-sometimes not even continuously-on incoming data, so we must exclude all configurations which evolve to a grazing or triple collision.

A more serious difficulty is that initial configurations can have more than one solution. Here is a simple example, complementary to that given by Lanford [6]. Place particles at positions $q_{i}, i=1,2, \ldots$ in a straight line so that

$$
\sum_{i=1}^{\infty}\left(q_{i+1}-q_{i}-a\right)<\infty . \quad\left(\text { Each } q_{i+1}-q_{i}>a .\right)
$$

All $p_{i}=0$. One solution is: all particles remain at rest forever. For a second solution, choose a number $v>0$ and for $i=1,2, \ldots$ let the particle at $q_{i}$ begin to move toward the particle at $q_{i-1}$ with speed $v$ at time

$$
t_{i}=(1 / v) \sum_{j=i}^{\infty}\left(q_{j+1}-q_{j}-a\right)
$$

and come to rest when it collides with the particle at $q_{i-1}$ at time $t_{i-1}=t_{i}+$ $v^{-1}\left(q_{i}-q_{i-1}-a\right)$.

It is easy to see that by allowing a moderate growth in speed as $i$ tends to infinity one can relax the requirements that the particles lie in a straight line and that the sum of the interparticle distances be finite.

To exclude pathological examples like this one and the one given by Lanford, we introduce in $\S 4$ the notion of a regular solution. Exponential growth of velocities (which occurs in Lanford's example) and too-close crowding of particles at infinity (as in this example) will be prohibited. It will be shown that any configuration of the infinite system has at most one regular solution, and that almost every configuration (in the sense of equilibrium probability) has a regular solution.

0.2. Notations, Conventions. We take our particles to have diameter $a$ and mass $m$, fixed positive numbers. Positions and momenta of particles are vectors $q, p$, respectively, of $\mathbb{R}^{v}$. Positions of $n$ particles are points $\boldsymbol{q}$ of $\mathbb{R}^{n v}$, which we think of as $n$-tuples $\left(q_{1}, \ldots, q_{n}\right)$ of points of $\mathbb{R}^{v}$; similarly for momenta.

A bounded Lebesgue-measurable subset $\Lambda$ of $\mathbb{R}^{v}$ has volume $|\Lambda|$.

In a set $\Omega$, with subset $A \subset \Omega, A^{c}=\Omega \backslash A$ is the complement of $A . I_{A}$ is the indicator function of $A$, i.e., the function on $\Omega$ which is 1 on $A$ and 0 on $A^{c}$.

Boltzmann factors involving the hard-sphere potential $\Phi$ reduce to indicators; they occur so frequently that we give them a name:

$$
J\left(q_{1}, q_{2}\right) \equiv \exp \left[-\beta \Phi\left(q_{1}-q_{2}\right)\right]\left(=I_{\left[\left|q_{1}-q_{2}\right| \geqq a\right]}\right) .
$$

Here $\left[\left|q_{1}-q_{2}\right| \geqq a\right]$ means the set of points $\left(q_{1}, q_{2}\right)$ of $\mathbb{R}^{2 v}$ satisfying $\left|q_{1}-q_{2}\right| \geqq a$. 


\section{Phase Space}

1.1. Definition. $\mathfrak{X}_{\infty}$, the phase space for infinitely many particles in $\mathbb{R}^{v}$, is the set of all functions $x$ from $\mathbb{R}^{v} \times \mathbb{R}^{v}$ to the non-negative integers such that for any bounded set $\Lambda \subset \mathbb{R}^{v}$,

$$
\sum_{(q, p) \in \Lambda \times \mathbb{R}^{v}} x(q, p)<\infty .
$$

1.2. An element $x$ of $\mathfrak{X}_{\infty}$ is called a locally finite configuration; $x(q, p)$ is the number of particles at the position $q$ having momentum $p$. For Lebesgue measurable $\Lambda \subset \mathbb{R}^{v}$ we define $\mathfrak{X}_{\Lambda}$, the space of locally finite configurations of particles in $\Lambda$, in the obvious way; thus we have the decomposition

$$
\mathfrak{X}_{\infty}=\mathfrak{X}_{\Lambda} \times \mathfrak{X}_{\Lambda^{c}}
$$

which simply says that a configuration consists of the particles inside $\Lambda$ and those outside $\Lambda$.

We agree to the following abuse of notation: to each configuration $x$ we associate the function on $\mathbb{R}^{v}$, also called $x$, defined by

$$
x(q)=\sum_{p \in \mathbb{R}^{v}} x(q, p) .
$$

This is a convenient device when only the positions of particles in a configuration matter.

1.3. $\mathfrak{X}_{\infty}$ has a topology in which two configurations are close if they are close on a compact set. Let $\mathscr{K}_{1}$ be the space of continuous real functions on $\mathbb{R}^{v} \times \mathbb{R}^{v}$ whose support, projected on the first factor, is compact. For each function $\varphi \in \mathscr{K}_{1}$ define a function $\hat{\varphi}$ on $\mathfrak{X}_{\infty}$ by

$$
\hat{\varphi}(x)=\sum_{(q, p) \in \mathbb{R}^{v} \times \mathbb{R}^{v}} x(q, p) \varphi(q, p) .
$$

The condition (1.1.1) on $x$ and the support properties of $\varphi$ guarantee that $\hat{\varphi}$ is finite-valued; we give $\mathfrak{X}_{\infty}$ the weakest topology making all functions $\hat{\varphi}$ continuous. It can be shown [4] that $\mathfrak{X}_{\infty}$ with this topology is a Polish space, that is, it has a countable dense subset and the topology comes from a metric in which $\mathfrak{X}_{\infty}$ is complete.

1.4. Definition. $\mathfrak{X}$, the space of configurations of hard spheres of diameter $a$, is the subset of $\mathfrak{X}_{\infty}$ consisting of configurations $x$ such that

a) $\sup _{q \in \mathbb{R}^{v}} \sum_{p \in \mathbb{R}^{v}} x(q, p) \leqq 1$,

b) $x(q)=1=x\left(q^{\prime}\right)$ and $q \neq q^{\prime}$ implies $\left|q-q^{\prime}\right| \geqq a$.

1.5. Hard-sphere configurations have at most one particle (centered) at any point of space, and any two of their particles are separated by a distance not less than $a$. $\mathfrak{X}$ is evidently"closed in $\mathfrak{X}_{\infty}$, hence is itself a Polish space.

There is a slight technical difficulty, because the product decomposition $\mathfrak{X}=\mathfrak{X}_{\Lambda} \times \mathfrak{X}_{\Lambda^{c}}$ for subsets $\Lambda$ of $\mathbb{R}^{v}$ no longer holds. We circumvent this by identifying $\mathfrak{X}$ with its image in the product $\mathfrak{X}_{A} \times \mathfrak{X}_{A^{c}}$, and taking measures on $\mathfrak{X}$ to be measures on the product which are zero on the complement of (the image of) $\mathfrak{X}$. 


\section{Gibbs States}

2.1. A Gibbs state is a measure on $\mathfrak{X}$ describing thermal equilibrium. Specifically, a Gibbs state for the hard-sphere potential $\Phi$, with inverse temperature $\beta$ and activity $z$, is a Borel probability measure $\mu$ on $\mathfrak{X}$ satisfying the Equilibrium Equations:

For every bounded Borel set $\Delta \subset \mathbb{R}^{v}$ and function $\varphi$ in $L^{1}(\mathfrak{X}, \mu)$

$$
\begin{aligned}
\int_{\mathfrak{X}} \varphi d \mu= & \sum_{n=0}^{\infty} \frac{z^{n}}{n !}\left(\frac{\beta}{2 \pi m}\right)^{n v / 2} \int_{\Delta^{n} \times \mathbb{R}^{n v}} \boldsymbol{d q} \boldsymbol{d} \boldsymbol{p} \prod_{i<j} J\left(q_{i}, q_{j}\right) \\
& \cdot \exp \left[-\beta \boldsymbol{p}^{2} /(2 m)\right] \int_{\mathfrak{X}_{\mathbb{R}^{\nu} \backslash \Delta}} \mu(d y) \prod_{\substack{1 \leqq i \leqq n \\
y(q)=1}} J\left(q_{i}, q\right) \cdot \varphi((\boldsymbol{q}, \boldsymbol{p}), y) .
\end{aligned}
$$

The Equilibrium Equations just determine the conditional distribution for $x$ in $\mathfrak{X}_{\Delta}$ given $y$ in $\mathfrak{X}_{\mathbb{R}^{v} \backslash \Delta}$.

In any Gibbs state $\mu$, the number of particles in a bounded Borel set $\Delta \subset \mathbb{R}^{\nu}$ -

$$
n_{\Delta}(x)=\sum_{(q, p) \in \Delta \times \mathbb{R}^{v}} x(q, p)
$$

is a random variable bounded a.s. $-\mu$ by a constant times $|\Delta|$ because of the hard core condition; in particular $\int_{\mathfrak{X}} n_{\Delta} d \mu$, the mean number of particles in $\Delta$, is bounded by const $|\Delta|$.

2.2. Maxwell Velocity Distribution. It follows from the equilibrium equations that the momenta in a Gibbs state are independent $v$-dimensional Gaussian random variables with mean 0 and variance $v m / \beta$. For each bounded Borel set $\Lambda \subset \mathbb{R}^{v}$ we introduce the useful random variable

$$
V_{\max }(x)=V_{\max }(x ; \Lambda)=0 \vee \sup \left\{|p| / m:(q, p) \in \Lambda \times \mathbb{R}^{v}, x(q, p)=1\right\} .
$$

$V_{\max }(\cdot ; \Lambda)$ is the speed of the fastest particle in $\Lambda$.

Let us first compute an elementary estimate for the conditional probability that $V_{\max }>\lambda>0$, given that there are $k$ particles in $\Lambda$. The probability that a particle has velocity less than $\lambda$ is

$$
\begin{aligned}
P(\lambda) & =(\beta / 2 \pi m)^{v / 2} \int_{\left\{p \in \mathbb{R}^{v:}|p| / m<\lambda\right\}} \exp \left[-\beta p^{2} / 2 m\right] d p \\
& =(\beta / 2 \pi m)^{v / 2} \frac{2 \pi^{v / 2}}{\Gamma(v / 2)} \int_{0}^{m \lambda} u^{v-1} \exp \left[-\beta u^{2} / 2 m\right] d u
\end{aligned}
$$

so that

$$
\mu\left(V_{\max }>\lambda \mid n_{\Lambda}=k\right)=1-[P(\lambda)]^{k} \leqq k(1-P(\lambda)) \leqq \text { const } \cdot k \cdot e^{-\gamma \lambda^{2}}
$$

for any $\gamma<m \beta / 2$.

Using this estimate, we have for any bounded Borel set $\Lambda$

$$
\begin{aligned}
\mu\left[V_{\max }>\lambda\right] & =\sum_{k} \mu\left(V_{\max }>\lambda \mid n_{\Lambda}=k\right) \mu\left[n_{\Lambda}=k\right] \\
& \leqq \text { const } e^{-\gamma \lambda^{2}} \sum_{k} k \mu\left[n_{\Lambda}=k\right] \leqq \text { const } \cdot e^{-\gamma \lambda^{2}}|\Lambda|
\end{aligned}
$$


since the sum over $k$ in the last line is just the expectation of $n_{\Lambda}$. It follows that $\exp \left[\gamma V_{\max }^{2}\right]$ is $\mu$-integrable for any $\gamma<m \beta / 2$, and

$$
\int_{\mathfrak{X}} \exp \left[\gamma V_{\max }^{2}(x ; \Lambda)\right] \mu(d x) \leqq M|\Lambda|
$$

with a constant $M$ which is independent of $\Lambda$.

2.3. Correlation Functions. Every Gibbs state $\mu$ has correlation functions defined by [8]

$$
\begin{aligned}
\varrho_{n}\left(q_{1}, \ldots, q_{n}\right) & =z^{n} \int_{\mathfrak{X}} \mu(d y) \exp \left[-\beta \sum_{i<j} \Phi\left(q_{i}-q_{j}\right)\right] \exp \left[-\beta \sum_{\substack{1 \leqq i \leqq n \\
y(q) \neq 0}} \Phi\left(q_{i}-q\right)\right] \\
& =z^{n} \prod_{i<j} J\left(q_{i}, q_{j}\right) \int_{\mathfrak{X}} \mu(d y) \prod_{\substack{1 \leqq i \leqq n \\
y(q) \neq 0}} J\left(q_{i}, q\right) .
\end{aligned}
$$
set, let

If $\mathscr{P}$ is some statement about points of $\mathbb{R}^{n v}$, and $\Lambda \subset \mathbb{R}^{v}$ is a bounded Borel

$$
E=\left\{\boldsymbol{q} \in \Lambda^{n}: q \text { satisfies } \mathscr{P}\right\} ;
$$

then $\int_{E} \varrho_{n}(q) d q$ is just the expectation value in the Gibbs state $\mu$ of the number of $n$-tuples of particles in $\Lambda$ satisfying $\mathscr{P}$.

From the definition one immediately deduces the basic inequality for correlation functions [8, Exercise 4.D]:

$$
\varrho_{n}(\boldsymbol{q}) \leqq z^{n} \prod_{1 \leqq i<j \leqq n} J\left(q_{i}, q_{j}\right) .
$$

\section{Probability Estimates}

3.1. Proposition.("Chain estimate"). Let $\mu$ be a Gibbs state for $\Phi$ with activity $z$. For any bounded Borel set $\Lambda$ of $\mathbb{R}^{v}$, any integer $N>1$ and any collection $\varepsilon_{1}, \varepsilon_{2}, \ldots, \varepsilon_{N-1}$ of positive numbers less than 1 , the probability that there are $N$ particles in $\Lambda$ at positions $q_{1}, \ldots, q_{N}$ satisfying $\left|q_{i+1}-q_{i}\right| \leqq a\left(1+\varepsilon_{i}\right)$ for $i=1,2, \ldots, N-1$, is less than

$$
N !|\Lambda| z^{N}\left[\frac{2 \pi^{v / 2}\left(2^{v}-1\right)}{v \Gamma(v / 2)} a^{v}\right]^{N-1} \prod_{i=1}^{N-1} \varepsilon_{i} .
$$

Proof. Let $X$ be the random variable which counts the number of $N$-tuples of particles in $\Lambda$ satisfying the stated condition; the probability to be estimated is $\mu[X \geqq 1]$ which is not more than $\int X d \mu$. This integral is given by an integral of the $N$-particle correlation function: put

Then

$$
\begin{aligned}
E= & \left\{\boldsymbol{q} \in \Lambda^{N}:\left|q_{i_{j+1}}-q_{i_{j}}\right| \leqq\left(1+\varepsilon_{j}\right) a \text { for } 1 \leqq j \leqq N-1,\right. \\
& \text { for some permutation } \left.i_{1}, \ldots, i_{N} \text { of }(1,2, \ldots, N)\right\} .
\end{aligned}
$$

$$
\int_{\mathfrak{X}} X d \mu=\int_{E} \varrho_{N}(\boldsymbol{q}) \boldsymbol{d q} .
$$

Therefore

$$
\int_{\mathfrak{X}} X d \mu \leqq N ! \int_{\left\{\boldsymbol{q} \in \Lambda^{N}:\left|q_{i}+1-q_{l}\right| \leqq\left(1+\varepsilon_{i}\right) a, 1 \leqq i \leqq N-1\right\}} \varrho_{N}(\boldsymbol{q}) \boldsymbol{d} \boldsymbol{q} .
$$


Insert the basic inequality for correlation functions (2.3.2) into this integral and change to difference variables $y_{i}=q_{i+1}-q_{i}, i=1,2, \ldots, N-1$; then

$$
\begin{aligned}
\int_{\mathfrak{X}} X d \mu & \leqq N ! z^{N} \int_{\Lambda} d q_{1} \prod_{i=1}^{N-1} \int_{a \leqq\left|y_{i}\right| \leqq a\left(1+\varepsilon_{i}\right)} d y_{i} \\
& =N ! z^{N} \prod_{i=1}^{N-1} \frac{2 \pi^{v / 2}}{v \Gamma(v / 2)} a^{v}\left(\left(1+\varepsilon_{i}\right)^{v}-1\right),
\end{aligned}
$$

and since $(1+\varepsilon)^{v}-1<\left(2^{v}-1\right) \varepsilon$ if $0<\varepsilon<1$, the bound (3.1.1) follows.

3.2. Proposition ("Free distance estimate"). Let $\mu$ be a Gibbs state for $\Phi$ with inverse temperature $\beta$, and let $T^{t}$ be a one-parameter group of transformations of $\mathfrak{X}$ leaving $\mu$ invariant and having piecewise continuous orbits. For bounded Borel sets $\Lambda \subset \mathbb{R}^{v}$ define

$$
V_{\max }(t)=V_{\max }\left(T^{t} x ; \Lambda\right)=\sup \left\{|p| / m:(q, p) \in \Lambda \times \mathbb{R}^{v}, T^{t} x(q, p)=1\right\}
$$

the speed of the fastest particle in $\Lambda$ at time $t$. For any positive number $\gamma$ less than $\beta m / 2$ there is a constant $M$ independent of $\Lambda$ such that for any $\tau>0, \lambda>0$ :

$$
\mu\left[\int_{0}^{\tau} V_{\max }(t) d t>\lambda\right]<M|\Lambda| \exp \left(-\gamma \lambda^{2} / \tau^{2}\right) .
$$

Proof. Let $0<\gamma<m \beta / 2$, and define the random variable

$$
U_{1}=\exp \left\{\gamma\left(\frac{1}{\tau} \int_{0}^{\tau} V_{\max }(t) d t\right)^{2}\right\} .
$$

Since $d t / \tau$ is a probability on $[0, \tau]$ and $\exp \left[\gamma(\cdot)^{2}\right]$ is convex, Jensen's inequality gives

$$
U_{1} \leqq \frac{1}{\tau} \int_{0}^{\tau} \exp \left[\gamma V_{\max }^{2}(t)\right] d t \equiv U_{2}
$$

Let us show that $U_{2}$ (and therefore $U_{1}$ ) is $\mu$-integrable. We have

$$
\begin{aligned}
\int_{\mathfrak{X}} U_{2} d \mu & =\int_{\mathfrak{X}} d \mu \frac{1}{\tau} \int_{0}^{\tau} d t \exp \left[\gamma V_{\max }^{2}(t)\right] \\
& =\frac{1}{\tau} \int_{0}^{\tau} d t \int_{\mathfrak{X}} d \mu \exp \left[\gamma V_{\max }^{2}(t)\right] \\
& =\frac{1}{\tau} \int_{0}^{\tau} d t \int_{\mathfrak{X}} d \mu \exp \left[\gamma V_{\max }^{2}(0)\right] \leqq M|\Lambda| .
\end{aligned}
$$

The last equality is asserted first, and is true because $\mu$ is invariant under $T^{t}$; then the middle equality follows by Tonelli's theorem. The inequality at the end is just (2.2.3), and $M$ is independent of $\Lambda$. Finally, the event $\left[\int_{0}^{\tau} V_{\max }(t) d t>\lambda\right]$ is identical to the event $\left[U_{1}>\exp \left(\gamma \lambda^{2} / \tau^{2}\right)\right]$, so the desired estimate follows from the Markov inequality.

3.3. Remark. A similar bound can be proven for $\mu\left[\sup _{0 \leqq t \leqq \tau} V_{\max }(t)>\lambda\right]$. Since this is not necessary for our results, we prove it in the appendix. 


\section{Partial Flows}

4.1. For integers $r>1$ we define the partial flow in the sphere of radius $r$ about the origin of $\mathbb{R}^{v}$, by placing a hard wall at $|q|=r$, fixing all particles not entirely inside, and letting particles inside move according to the elastic reflection law.

For some configurations this prescription leads to triple collisions (simultaneous collisions of three or more particles) or grazing collisions (contacts with zero momentum transfer), beyond which trajectories do not depend smoothly, or not even continuously, on initial data, or perhaps are not even definable without ambiguity.

The problem of existence of partial flows for all time has been solved in [1] and we summarize the results here. Let

$$
\Lambda(r)=\left\{q \in \mathbb{R}^{v}:|q| \leqq r-a / 2\right\}
$$

Particles of a configuration $x$ which are in $\Lambda(r)$ are entirely inside the sphere of radius $r$ centered at the origin of $\mathbb{R}^{v}$; they suffer elastic collisions at the boundary, which consists of the hard wall at $|q|=r$, together with bumps made by the fixed particles in the annular region

$$
\Delta(r)=\left\{q \in \mathbb{R}^{v}: r-a / 2<|q|<r+a / 2\right\} .
$$

Any two configurations whose restrictions to $\Lambda(r) \cup \Delta(r)$ are the same, have the same motion in the $r^{\text {th }}$ partial flow.

4.2. Definition. $x \in \mathfrak{X}$ is a collision point if there exist $q, q^{\prime} \in \mathbb{R}^{v}$ with $\left|q-q^{\prime}\right|=a$ and $x(q)=x\left(q^{\prime}\right)=1 . x \in \mathfrak{X}$ has pairwise collisions if $x$ is a collision point and for every $q, q^{\prime} \in \mathbb{R}^{v}$ with $\left|q-q^{\prime}\right|=a$ and $x(q)=x\left(q^{\prime}\right)=1$ there is no $q^{\prime \prime}$ distinct from $q$ and $q^{\prime}$ with $\left|q^{\prime \prime}-q\right|=a$ or $\left|q^{\prime \prime}-q^{\prime}\right|=a$ and $x\left(q^{\prime \prime}\right)=1$. $x \in \mathfrak{X}$ has non-grazing collisions if whenever $x(q, p)=x\left(q^{\prime}, p^{\prime}\right)=1$ with $\left|q-q^{\prime}\right|=a$, one has $\left\langle p-p^{\prime}, q-q^{\prime}\right\rangle \neq 0$.

Let $\mathscr{S}$ be the subset of $\mathfrak{X}$ consisting of configurations with no collisions, or collision points with pairwise, nongrazing collisions. Then $\mathscr{S}$ is a dense $G_{\delta}$, with full measure in every Gibbs state.

Now let $T_{r}^{t}$ be the transformation of $\mathfrak{X}$ which shifts a configuration $t$ units along its trajectory under the flow in $\Lambda(r)$, wherever the latter is defined. The methods of [1, Chapter II] may be applied to show that every $T_{r}$ is defined for all time almost everywhere; we summarize these results in the next Proposition, which shows that the estimates on the $T_{r}$ to be proven in the remainder of this Chapter are not vacuous.

The estimates we shall prove have a wider application: they will show that any flow on $\mathfrak{X}$ which solves the equations of motion and leaves equilibrium states invariant, must have orbits with very good regularity properties.

4.3. Proposition.a) Let $S^{t}$ be a one-parameter group of transformations of $\mathfrak{X}$ given by solutions of the equations of motion, and leaving all Gibbs states invariant. Then there is a dense $G_{\delta}$ subset of $\mathfrak{X}$, of full measure in every Gibbs state, consisting of configurations whose orbits lie entirely in $\mathscr{S}$, and which have only finitely many collisions in any $\Lambda(r)$ during any finite t-interval.

b) There is a dense $G_{\delta}$ subset $\tilde{\mathfrak{X}}$ of $\mathfrak{X}$, of full measure in every Gibbs state, on which every $T_{r}^{t}$ is defined for all $t$; every orbit lies entirely in $\mathscr{S}$, and has only finitely 
many collisions during any bounded interval of time. Every $T_{r}^{t}$ leaves all Gibbs states invariant.

For the proof, we refer to [1]. In particular, the last statement of b) is proven by conditioning on $\Lambda^{c}(r)$ and using the Equilibrium Equations (2.1.1) and the fact, proven in [1, Chapter II] that Liouville's theorem holds for the flow in $\Lambda(r)$.

4.4. The estimates of the following sections will hold both for the partial flows and for any equilibrium-preserving global flow. Thus for each integer $r>1$ let

$$
x_{r}(t)=T_{r}^{t} x \quad(x \in \tilde{\mathfrak{X}})
$$

or

$$
x_{r}(t)=S^{t} x
$$

with $S^{t}$ satisfying the conditions of 4.3 a).

Choose two numbers $\alpha>5 / 2$ and $c>0$. Put

$$
\eta=3 \alpha / 5
$$

and choose $\omega$ in the open interval $(2 \alpha / 5,3 \alpha / 5-1 / 2)$; then

$$
1<2 \alpha / 5=\alpha-\eta<\omega<\eta-1 / 2
$$

and

$$
2(\eta-\omega)>1 .
$$

Let $r$, an integer greater than 1 , be large enough that

$$
\begin{aligned}
& 4(\log r)^{-\omega}<a, \\
& 8(\log r)^{-\omega}<c(\log r)^{\alpha-\eta}
\end{aligned}
$$

and define

$$
\begin{aligned}
N & =N(r)=\left[(2 a)^{-1} c(\log r)^{\alpha-\eta}\right], \\
\tau & =\tau(r)=(\log r)^{-\eta} .
\end{aligned}
$$

Let $\mu$ be a Gibbs state for $\Phi$ with inverse temperature $\beta$ and activity $z$, and let $\gamma<\beta m / 2$ and $M$ be the constants for which 3.2 holds. Finally, let $C_{0}$ be $z^{N /(N-1)}$ times the expression in brackets in (3.1.1).

\section{Define}

$$
V_{\max }(t)=V_{\max }\left(x_{r}(t) ; \Lambda(r)\right)=\sup \left\{|p| / m:(q, p) \in \Lambda(r) \times \mathbb{R}^{v}, x_{r}(t)(q, p)=1\right\} .
$$

Define $A_{r}(t)$ to be the event that either

Or

a) $\int_{t}^{t+\tau} V_{\max }(s) d s>\frac{1}{2}(\log r)^{-\omega}$

b) $x_{r}(t)$ has $N(r)$ particles in $\Lambda(r)$ at positions $q_{1}, \ldots, q_{N}$ satisfying

$$
\left|q_{i+1}-q_{i}\right| \leqq a+4(\log r)^{-\omega}, \quad i=1,2, \ldots, N-1 .
$$

$1 \quad[\lambda]$, "ceiling of $\lambda$ ", is the smallest integer greater than $\lambda$ 


\subsection{Lemma.}

$$
\mu A_{r}(0) \leqq|\Lambda(r)|\left\{M \exp \left[-(\gamma / 4)(\log r)^{2(\eta-\omega)}\right]+N(r) !\left(4 c_{0} a^{-1}(\log r)^{-\omega}\right)^{N(r)-1}\right\} .
$$

Proof. Immediate. Use 3.1 with $\varepsilon_{i}=4 a^{-1}(\log r)^{-\omega}$ [note (4.4.4)]; and use 3.2 with $\tau=\tau(r)=(\log r)^{-\eta}$ and $\lambda=(1 / 2)(\log r)^{-\omega}$.

Now let $t_{0}>0$ and put

$$
K\left(t_{0}\right)=\left[t_{0}(\log r)^{\eta}\right] .
$$

The next lemma is an immediate consequence of the invariance of $\mu$ under $T_{r}^{t}$ or $S^{t}$.

4.6. Lemma. Put $B_{r}\left(t_{0}\right)=\bigcup_{j=-K}^{K-1} A_{r}(j \tau)$. Then $\mu B_{r}\left(t_{0}\right) \leqq 2 K \mu A_{r}(0)$.

4.7. Proposition.For every $x$ in a Borel set $\hat{\mathfrak{X}}$ of $\mathfrak{X}$ of full measure in every Gibbs state, and every $t_{0}$ positive there is an $r_{0}=r_{0}\left(x, t_{0}\right)$ such that if $r>r_{0}$ :

a) there is no subinterval $I \subset\left[-t_{0}, t_{0}\right]$ of length $\tau(r)$ for which

$$
\int_{I} V_{\max }\left(x_{r}(t)\right) d t>(\log r)^{-\omega}
$$

and

b) at no time $t,-t_{0} \leqq t \leqq t_{0}$, does $x_{r}(t)$ have $N(r)$ particles in $\Lambda(r)$ at positions $q_{1}, \ldots, q_{N(r)}$ such that

$$
\left|q_{i+1}-q_{i}\right| \leqq a+2(\log r)^{-\omega}, \quad i=1,2, \ldots, N-1 .
$$

Proof. For positive integers $k$ let $\mathfrak{X}_{k}=\left[B_{r}(k) \text { i.o. }\right]^{c}$. We show $\sum_{r} \mu B_{r}(k)<\infty$ for any Gibbs state $\mu$, so that $\mu \mathfrak{X}_{k}=1$ by the Borel-Cantelli lemma.

From 4.5 and 4.6 we have a bound for $\mu B_{r}(k)$ which we write as a sum of two terms. The first is

$$
\begin{aligned}
& K(k)|\Lambda(r)| M \exp \left[-(\gamma / 4)(\log r)^{2(\eta-\omega)}\right] \\
& \quad \leqq \operatorname{const}(\log r)^{\eta} \cdot r^{\nu} \exp \left[-(\gamma / 4)(\log r)^{2(\eta-\omega)}\right]
\end{aligned}
$$

and since $2(\eta-\omega)>1(4.4 .3)$ this term is summable in $r$.

The second term in the bound for $\mu B_{r}(k)$ coming from 4.5 .6 is

$$
\begin{aligned}
& K(k)|\Lambda(r)| N(r) !\left(4 c_{0} a^{-1}(\log r)^{-\omega}\right)^{N(r)-1} \\
& \quad \leqq \operatorname{const}(\log r)^{\eta} r^{v} N(r) ! \exp [-\omega(N(r)-1) \log \log r+O(N(r))] .
\end{aligned}
$$

Now use Stirling's formula and the definition (4.4.6) to write

$$
N(r) !=\exp [(\alpha-\eta) N(r) \log \log r+O(N(r))] .
$$

Then the term in question is bounded by

$$
\text { const }(\log r)^{\eta} r^{v} \exp \left[c(2 a)^{-1}(\alpha-\eta-\omega)(\log r)^{\alpha-\eta} \log \log r+O(\log r)^{\alpha-\eta}\right]
$$

and the exponential goes to zero more rapidly than any inverse power of $r$ because $(\alpha-\eta-\omega)<0$ and $\alpha-\eta>1$ by (4.4.2). 
This completes the proof that $\sum_{r} \mu B_{r}(k)<\infty$. Thus for each $k, \mu \mathfrak{X}_{k}=1$, where $\mathfrak{X}_{k}$ consists of phase points which are in $B_{r}(k)$ for at most finitely many $r$.

Now if $x \in\left[B_{r}(k)\right]^{c}$, there is no interval $I$ of length $\tau(r)$ in $[-k, k]$ for which the free distance $\int_{I} V_{\max }\left(x_{r}(t)\right) d t$ exceeds $(\log r)^{-\omega}$. For if there were, we could choose $j,-K(k) \leqq j \leqq K(k)-1$, so that

$$
\int_{j \tau}^{(j+1) \tau} V_{\max }\left(x_{r}(t)\right) d t \geqq \int_{I \cap[j \tau,(j+1) \tau]} V_{\max }\left(x_{r}(t)\right) d t \geqq(1 / 2)(\log r)^{-\omega}
$$

which is impossible because $x \in\left[A_{r}(j \tau)\right]^{c}$.

Moreover, if at any time $t,-k \leqq t \leqq k, x_{r}(t)$ had $N(r)$ particles in $\Lambda(r)$ at positions $q_{1}, \ldots, q_{N}$ with $\left|q_{i+1}-q_{i}\right|<a+2(\log r)^{-\omega}$ for $i=1,2, \ldots, N-1$, then by choosing $j$ so that $j \tau$ is the integral multiple of $\tau$ nearest to $t$, and letting $q_{i}(s)$ denote the position at time $s$ of the particle of $x_{r}$ which is at $q_{i}$ at time $t$, we would derive

$$
\begin{gathered}
\left|q_{i+1}(j \tau)-q_{i}(j \tau)\right| \leqq\left|q_{i+1}(j \tau)-q_{i+1}(t)\right|+\left|q_{i+1}(t)-q_{i}(t)\right|+\left|q_{i}(t)-q_{i}(j \tau)\right| \\
<(\log r)^{-\omega}+\left(a+2(\log r)^{-\omega}\right)+(\log r)^{-\omega}=a+4(\log r)^{-\omega}
\end{gathered}
$$

which is again impossible because $x \in\left[A_{r}(j \tau)\right]^{c}$.

To complete the proof, put $\hat{\mathfrak{X}}=\bigcap_{k} \mathfrak{X}_{k}$. Then $\mu \hat{\mathfrak{X}}=1$ for every Gibbs state $\mu$, and every $x$ in $\hat{\mathfrak{X}}$ satisfies a) and $b$ ).

4.8. Definition. A mapping $\xi: \mathbb{R} \rightarrow \mathfrak{X}$ is a regular solution of the equations of motion if

a) $\xi$ is a solution of the equations of motion, with $\xi(t) \in \mathscr{S}$ (see 4.2) for all $t$, and only finitely many collisons occur in any $\Lambda(r)$ during any bounded $t$-interval; and

b) for any $t>0$ there is an $r_{1}=r_{1}(\xi, t)$ such that if $r>r_{1}$ then

i) for every subinterval $I$ of $[-t, t]$ of length $\tau(r)$,

$$
\int_{I} V_{\max }(\xi(s) ; \Lambda(r)) d s \leqq(\log r)^{-\omega},
$$

ii) at no time during $[-t, t]$ is there a chain of $N(r)$ particles in $\Lambda(r)$ at positions $q_{1}, \ldots, q_{N}$ such that

$$
\left|q_{i+1}-q_{i}\right|<a+(\log r)^{-\omega}, \quad i=1,2, \ldots, N-1 .
$$

The fact that every $x_{r}(t)$ could be $S^{t} x$ for an equilibrium-preserving flow $S^{t}$ which solves the equations of motion, allows us to combine 4.3 a) and 4.7 to get that almost every orbit is regular.

4.9. Proposition. Let $S^{t}$ be a one-parameter group of transformations of $\mathfrak{X}$ given by solutions of the equations of motion, which leave all Gibbs states invariant. Then the set of phase points whose orbits are regular solutions has probability one in every Gibbs state. 


\section{Dynamics of the Infinite System}

5.1. Proposition. Let $x \in \hat{\mathfrak{X}}$ and $t_{0}>0$; suppose that $x\left(q_{0}, p_{0}\right)=1$ and take $r>r_{0}\left(x, t_{0}\right)$ $\left(r_{0}\right.$ given by 4.7) so large that

$$
\left|q_{0}\right|+c\left(1+t_{0}^{2}\right)^{1 / 2}(\log 2 r)^{\alpha}<r / 2 .
$$

Put $x_{r}(t)=T_{r}^{t} x$, and denote by $q^{(r)}(t), p^{(r)}(t)$ the position and momentum in $x_{r}(t)$ of the particle which is at $(q, p)$ at time zero (at collisions take all momenta to be incoming). Then for $j=1,2, \ldots, r$ and for all $t, 0 \leqq t \leqq t_{0}$,

$$
q_{0}^{(r+j)}(t)=q_{0}^{(r)}(t) \quad \text { and } \quad p_{0}^{(r+j)}(t)=p_{0}^{(r)}(t)
$$

Remark. This proposition asserts that the effect of particles outside $\Lambda(r)$ "proagates with finite speed" when the hard-wall boundary is removed.

Proof. Let $r^{\prime}$ be any $r+j, j=1,2, \ldots, r$, and say that a particle of $x$ in $\Lambda\left(r^{\prime}\right)$ gets marked at time $t$ if $t$ is the infimum of the set of times for which the particle's $T_{r^{\prime}}$ motion differs from its $T_{r}$ motion. For example, all particles in $\Lambda\left(r^{\prime}\right) \backslash \Lambda(r)$ are marked at time zero, except those at rest or undergoing a collision which brings them to rest.

Take any particle of $x$ which is inside $\Lambda(r)$ at time zero, and which is marked at some time $t_{1}, 0 \leqq t_{1} \leqq t_{0}$. Call this particle 1 . We construct a finite chain of particles reaching from particle 1 to the boundary of $\Lambda(r)$, by the following procedure.

Step 1. Set $k:=1$. Go to Step 2.

Step 2. If particle $k$ gets marked by reaching the boundary of $\Lambda(r)$ the chain terminates with particle $k$. Otherwise go to Step 3 .

Step 3. Since particle $k$ is in the interior of $\Lambda(r)$ at time $t_{k}$ it gets marked by colliding under either $T_{r}$ or $T_{r^{\prime}}$ with a particle which is already marked. Call the latter particle $k+1$; it was marked at some time $t_{k+1}$ with $0 \leqq t_{k+1}<t_{k}$. Go to Step 4.

Step 4. Increment $k$ by 1 and return to Step 2 .

This construction terminates, resulting in a chain of finite length, because only finitely many collisions occur in $\Lambda(r)$ under $T_{r}$ or $T_{r^{\prime}}$ during $\left[0, t_{0}\right]$. We have, say, $n$ particles in the chain, and $\left|q_{n}\left(t_{n}\right)\right|=r$. Notice that since a particle behaves the same under $T_{r}$ and $T_{r^{\prime}}$ until it gets marked, we have $q_{i}^{(r)}\left(t_{i}\right)=q_{i}^{\left(r^{\prime}\right)}\left(t_{i}\right)$ for $i=1,2, \ldots, n$.

We are interested in how far a particle can be from the boundary when it gets marked. Suppose that $r-\left|q_{1}\left(t_{1}\right)\right|>c\left(1+t_{0}^{2}\right)^{1 / 2}(\log 2 r)^{\alpha}$. Note first that $\left|q_{n}\left(t_{n}\right)-q_{1}\left(t_{1}\right)\right|>r-\left|q_{1}\left(\dot{t}_{1}\right)\right|$. Divide the interval $\left[0, t_{1}\right]$ into subintervals of length $\tau(2 r)=(\log 2 r)^{-\eta}$ [preserving the notations of $\left.(4.4 .1-7)\right]$.

Now for $k=0,1,2, \ldots,\left[t_{1} / \tau\right]$ let $i_{k}$ be the index of the first particle of the chain to be marked after time $k \tau$. Then there must be an index $k$ for which

$$
\begin{aligned}
\mid q_{i_{k+1}}((k+1) \tau)- & q_{i_{k}}(k \tau) \mid>(3 / 4) c(\log 2 r)^{\alpha-\eta} . \\
\text { (Else }\left|q_{n}\left(t_{n}\right)-q_{1}\left(t_{1}\right)\right| & \leqq \sum_{k}\left|q_{i_{k+1}}((k+1) \tau)-q_{i_{k}}(k \tau)\right| \\
& \leqq\left[t_{1} / \tau\right](3 / 4) c(\log 2 r)^{\alpha-\eta}<c\left|t_{1}\right|(\log 2 r)^{\alpha} \\
& <r-\left|q_{1}\left(t_{1}\right)\right| \text { which is impossible .) }
\end{aligned}
$$


But during the interval $[k \tau,(k+1) \tau]$ no particle travels a distance greater than $(\log r)^{-\omega}$ in either its $T_{r}$ or its $T_{r^{\prime}}$ motion; therefore at time $k \tau$ there must be a chain of at least

$$
(3 / 4) c(\log 2 r)^{\alpha-\eta} /\left(a+2(\log r)^{-\omega}\right)>c(2 a)^{-1}(\log r)^{\alpha-\eta} \geqq N(r)
$$

particles in $\Lambda(r)$, each at a distance smaller than $2(\log r)^{-\omega}$ from the next. But because $x \in \hat{\mathfrak{X}}$ this is impossible.

5.2. Theorem. For every $x \in \hat{\mathfrak{X}}$ the limit $\lim _{r \rightarrow \infty} T_{r}^{t} x$ exists in the $\mathfrak{X}$-topology uniformly on compact $t$-intervals, and for each fixed $t$ defines a Borel-measurable transformation of $\hat{\mathfrak{X}}$ into $\mathfrak{X}$ leaving all Gibbs states invariant.

Proof. Let $x \in \hat{\mathcal{X}}$ and number its particles in the order of their distance from the origin of $\mathbb{R}^{v}$. Let $t_{0}>0$; for each $i=1,2, \ldots$ we apply 5.1 repeatedly to show the existence of an $r_{i}$ so that the $i^{\text {th }}$ particle has the same motion under any $T_{r^{\prime}}, r^{\prime}>r_{i}$, throughout $\left[-t_{0}, t_{0}\right]$.

Now let $\varphi$ be a real function on $\mathbb{R}^{v} \times \mathbb{R}^{v}$, the projection of the support of $\varphi$ on the first factor being a compact set $K$ (see 1.3). Take $r>r_{0}\left(x, t_{0}\right)\left(r_{0}\right.$ given by 4.7) so large that all translates of $K$ by distances less than $c\left(1+t_{0}^{2}\right)^{1 / 2}(\log r)^{\alpha}$ lie inside $\Lambda(r / 4)$. Then $T_{r^{\prime}}^{t} x_{K \times \mathbb{R} v}$ is independent of $r^{\prime}>r$ for all $t$ in $\left[-t_{0}, t_{0}\right]$, and it follows that $\hat{\varphi}\left(x_{r^{\prime}}(t)\right)$ is also independent of $r^{\prime}$, so long as $r^{\prime}>r$, for every $t$ in $\left[-t_{0}, t_{0}\right]$. Since functions of the form $\hat{\varphi}$ define the topology of $\mathfrak{X}, T_{r}^{t} x$ converges as $r \rightarrow \infty$ and the convergence is uniform on compact intervals in $t$.

The transformation $T^{t}$, defined for $x \in \hat{\mathfrak{X}}$ by $T^{t}=\lim _{r \rightarrow \infty} T_{r}^{t} x$, being the pointwise limit of Borel-measurable transformations, is itself Borel measurable; each orbit is a regular solution of the equations of motion for the infinite configuration.

We now prove the invariance of Gibbs states under $T^{t}$. Let $\mu$ be a Gibbs state, $f$ a bounded continuous function on $\mathfrak{X}$. For each integer $r>1$ define

$$
\begin{aligned}
f_{r}(x) & =f\left(T_{r}^{-t} x\right) \\
f_{\infty}(x) & =f\left(T^{-t} x\right) .
\end{aligned}
$$

Since $T_{r}^{-t} \rightarrow T^{-t}$ a.s. $-\mu$, we have $f_{r} \rightarrow f_{\infty}$ a.s. $-\mu$, so $f_{\infty}$ is $\mu$-measurable. Moreover the $f_{r}, 1<r \leqq \infty$, are uniformly bounded by $\|f\|_{\infty}$, so by the Dominated Convergence Theorem $\int f_{\infty} d \mu=\lim _{r \rightarrow \infty} \int f_{r} d \mu=\int f d \mu$, the last equality holding because $\mu$ is invariant under each $T_{r}^{t}$. This completes the proof, for a Borel probability measure on a Polish space is uniquely determined by the integrals of bounded continuous functions with respect to it [9].

Remark. Since $T_{r}^{t}$ converges almost surely in any Gibbs state $\mu$ to $T^{t}$, it follows that it converges in probability, and almost uniformly. The proof is exactly as in the theory of functions of a real variable, and since the fact will not be needed in the sequel, we omit it.

5.3. Theorem.(Uniqueness of regular solutions). For any $x \in \mathfrak{X}$ there is at most one regular solution $\xi: \mathbb{R} \rightarrow \mathfrak{X}$ of the equations of motion such that $\xi(0)=x$.

Proof. Let $\xi^{\prime}, \xi^{\prime \prime}$ be any regular solutions with $\xi^{\prime}(0)=\xi^{\prime \prime}(0)=x$. We write $\left(q^{\prime}(t), p^{\prime}(t)\right)$ [resp. $\left.\left(q^{\prime \prime}(t), p^{\prime \prime}(t)\right)\right]$ for the position and momentum in $\xi^{\prime}(t)$ [resp. $\left.\xi^{\prime \prime}(t)\right]$ of the particle of $x$ which has position $q$, momentum $p$. 
If $\xi^{\prime}$ is not identical to $\xi^{\prime \prime}$, we may suppose there is a particle $q_{1}$ and a time $t_{1}>0$ (since a time-reversed solution is a solution), so that $t_{1}=\inf \left\{t>0: q_{1}^{\prime}(t) \neq q_{1}^{\prime \prime}(t)\right\}$. Let $r$ be any integer larger than the maximum of $\left|q_{1}\right|+a, r\left(\xi^{\prime}, t_{1}\right), r\left(\xi^{\prime \prime}, t_{1}\right)$, the last two numbers coming from the definition $4.8 \mathrm{~b}$ ).

As in the proof of 5.1 say that a particle of $x$ at $q$ is marked at time $t$ if

(i) $t=0$ and $|q| \geqq r$ or

(ii) $t_{1} \geqq t>0$ and $t=\inf \left\{s: q^{\prime}(s) \neq q^{\prime \prime}(s)\right.$ or $\left.\left|q^{\prime}(s)\right| \geqq r\right\}$.

In particular $q_{1}$ is marked at time $t_{1}$. Use the fact that $\xi^{\prime}, \xi^{\prime \prime}$ have only finitely many collisions in $\Lambda(r)$ during $\left[0, t_{1}\right]$ to construct a chain of particles initially at $q_{1}, \ldots, q_{n}$ and corresponding times $t_{1}>t_{2}>\ldots>t_{n} \geqq 0$ so that for $i=1,2, \ldots, n-1$, $q_{i}$ is marked at time $t_{i}$ by collision with $q_{i+1}$ [in either $\xi^{\prime}\left(t_{i}\right)$ or $\left.\xi^{\prime \prime}\left(t_{i}\right)\right]$, and $\left|q_{n}^{\prime}\left(t_{n}\right)\right|=r$. (If this construction did not reach the boundary of $\Lambda(r)$ we would find a particle which before its first collision moved differently for $\xi^{\prime}$ and $\xi^{\prime \prime}$, which is impossible since both are solutions.)

The same argument as that of 5.1 now shows $r-\left|q_{1}\left(t_{1}\right)\right|<c\left(1+t_{1}^{2}\right)^{1 / 2}(\log r)^{\alpha}$, since the velocity bound and chain condition 4.8 (b) for regular solutions must hold. Therefore

$$
\left|q_{1}\right|>r-c\left(1+t_{1}^{2}\right)^{1 / 2}(\log r)^{\alpha}-t_{1}(\log r)^{-\omega} / \tau(r),
$$

but $r>\max \left\{\left|q_{1}\right|+a, r\left(\xi^{\prime}, t_{1}\right), r\left(\xi^{\prime \prime}, t_{1}\right)\right\}$ was arbitrary. The proof is complete.

5.4. Corollary. Let $S^{t}$ be a one-parameter group of transformations of $\mathfrak{X}$ into itself given by translating along solutions of the equations of motion, which leaves all Gibbs states invariant. Then for all $x$ in the complement of a set which is null for every Gibbs state, $S^{t} x=T^{t} x$ for all $t$.

Proof. Immediate from 4.9 and 5.3.

This result shows that although non-regular solutions exist, they cannot be pieced together to make a measure-preserving flow.

$\left\{T^{t}\right\}$ can be defined on a set possibly larger than $\hat{\mathfrak{X}}$ : if $y=T^{s} x \notin \hat{\mathfrak{X}}$ define $T^{t} y$ to be $T^{t+s} x$ for all $t$. This definition is unambiguous by the uniqueness of regular solutions, and it extends $\left\{T^{t}\right\}$ to a subset of $\mathfrak{X}$ which is invariant under the flow; thus $\left\{T^{t}\right\}$ becomes a group.

The time evolution in $\mathfrak{X}$ is not continuous: momenta change discontinuously at collisions. For single configurations we obtain a continuous time-evolution by identifying incoming and outgoing momenta at collision points. Since all orbits lie in the set $\mathscr{S}$ of 4.2 , of configurations with either no collisions or only pairwise, non-grazing collisions, it is enough to perform this identification in $\mathscr{S}$.

5.5. Definition. The relation $x \sim y$ on $\mathscr{S}$ means $x=y$ or

a) $x(q)=y(q)$ for all $q$ in $\mathbb{R}^{v}$;

b) any non-colliding particle of $x$ and $y$ has the same momentum in both configurations; and

c) if $x$ and $y$ have colliding particles at $q$ and $q^{\prime}$ with $\left|q-q^{\prime}\right|=a$, then the momenta, denoted by $p x, p^{\prime} x$ and $p y, p^{\prime} y$ respectively, either are the same in both configurations, or are obtained from each other by the elastic reflection law.

It is easy to see that $\sim$ is an equivalence relation, and we write $[x]$ for the equivalence class of $x \in \mathscr{S}$. It is now an elementary exercise in point-set topology to verify the next proposition. 
5.6. Proposition. For $x \in \mathscr{S},[x]$ is either finite or uncountable compact and perfect. Define $\Omega=\mathscr{S} / \sim$ to be the space of equivalence classes with the quotient topology; then the natural projection $P: \mathscr{S} \rightarrow \Omega$ is closed. By [2, Chapter IX, $\S 6$, Exercise 9 a)] there is $a G_{\delta}$ (hence Polish) subset of $\mathscr{S}$ which meets every equivalence class in exactly one point, so that $\Omega$, being the one-to-one continuous image of a Polish space, is a Lusin space. Any function $\hat{\varphi}$ in the family of 1.3 defining the topology of $\mathfrak{X}$, which is constant on equivalence classes, can be factored through the natural projection $P$ to define a continuous function on $\Omega$; the topology on $\Omega$ is the weakest topology making all such functions continuous.

Passing to the quotient now makes regular solutions continuous. There is another dividend as well; to formulate it, we define $T: \mathbb{R} \times P \hat{\mathfrak{X}} \rightarrow \Omega$ as follows. If $y \in P \hat{\mathfrak{X}}$ we take $x \in P^{-1} y$ to be the (unique) representative of its equivalence class such that all momenta of colliding particles are incoming. We define

$$
T(t, y)=P T^{t} x
$$

for all $t$.

5.7. Theorem. For any regular solution $\xi: \mathbb{R} \rightarrow \mathfrak{X}$ of the equations of motion, the mapping $P \circ \xi: \mathbb{R} \rightarrow \Omega$ is continuous. The mapping $T: \mathbb{R} \times P \hat{\mathfrak{X}} \rightarrow \Omega$ is Borel-measurable on the product space.

Proof. It is again elementary to verify the first statement. For the second, we have that (i) for fixed $x$, the mapping $t \mapsto T(t, x)$ is continuous, and (ii) for fixed $t$, the map $x \mapsto T(t, x)$ is Borel. The result follows from a theorem of [3].

Acknowledgement. I would like to thank O.E. Lanford III for posing this problem in the first place, and for much helpful advice as the work progressed.

\section{Appendix}

A.1. To prove existence and uniqueness of regular solutions it is enough to estimate the probability that the time-integral of $V_{\max }$ be large. Here we bound the supremem over an interval of $V_{\max }(t)$; analogous results have been obtained for other interactions $[7,10,11]$, but the methods do not extend to the pure hard-sphere case.

A.2. Proposition. Let $\mu$ be a Gibbs state for $\Phi$ with inverse temperature $\beta$ and activity $z$. Let $0<\gamma<m \beta / 2, \tau>0, \lambda>0$. For each positive integer $r$ put

$$
E_{r}(\tau)=\left\{x \in \mathfrak{X}: \sup _{0 \leqq t \leqq \tau} V_{\max }\left(T_{r}^{t} x ; \Lambda(r)\right)>\lambda\right\} .
$$

Then $\mu E_{r}(\tau) \leqq \operatorname{const}(1+\tau)|\Lambda(r)| \exp \left(-\gamma \lambda^{2}\right)$.

Proof. $E_{r}(\tau) \subset F_{0}+F_{\tau}$, where

$$
F_{0}=\left[V_{\max }(0)>\lambda\right], F_{t}=F_{0}^{c} \cap\left[\sup _{0<s \leqq t} V_{\max }(s)>\lambda\right] .
$$

First, (2.2.2) gives $\mu F_{0} \leqq$ const $|\Lambda(r)| \exp \left(-\gamma \lambda^{2}\right)$. Second, we put $P(t)=\mu F_{t}$ and estimate $P(\tau)$. 
For any $t$ positive and $\delta>0$ small, $P(t+\delta)-P(t)$ is the probability that $V_{\max }(t) \leqq \lambda$ and during the interval $[t, t+\delta]$ the speed of some particle in $\Lambda(r)$ is boosted above $\lambda$ by collisions. Such a boost requires either (1) two particles, the sum of whose kinetic energies is greater than $m \lambda^{2} / 2$, separated by a distance at most $a+2 \lambda \delta$, or (2) a particle which can undergo at least two collisions with other particles during $[t, t+\delta]$.

Take $\delta<a /(3 \lambda)$.

Estimate the probability of (1) by 3.1 and the Maxwell distribution; choose $\gamma^{\prime}$ so that $\gamma<\gamma^{\prime}<m \beta / 2$, so the probability of having two particles which can collide and produce a particle with speed greater than $\lambda$ is less than

$$
2 !|\Lambda(r)| z^{2} C_{0}(2 \lambda \delta / a) \cdot \text { const } \exp \left(-\gamma^{\prime} \lambda^{2}\right) \leqq \text { const } \delta|\Lambda| e^{-\gamma \lambda^{2}} .
$$

Next, if $V_{\max }(t) \leqq \lambda$, the velocity of a particle following the first collision after time $t$ is at most $2^{1 / 2} \lambda$; thus the first particle to suffer at least two collisions in time $\delta$, must have two particles within $a+2 \cdot 2^{1 / 2} \lambda \delta$ of it. Apply 3.1 again to bound the probability of this event by

$$
3 !|\Lambda(r)| z^{3} C_{0}^{2}\left(8 \lambda^{2} \delta^{2} / a^{2}\right) .
$$

Now combine (A.2.1) and (A.2.2) to see that

$$
(1 / \delta)(P(t+\delta)-P(t)) \leqq \text { const }|\Lambda(r)| \exp \left(-\gamma \lambda^{2}\right)+O(\delta)
$$

as soon as $\delta$ is sufficiently small. Thus $P(t)$ is absolutely continuous; we also have a bound on its derivative, and it follows by integration that

$$
P(\tau)=\mu F_{\tau} \leqq \text { const } \cdot \tau \cdot|\Lambda(r)| \exp \left(-\gamma \lambda^{2}\right)
$$

and the proof is terminated.

A.3. Theorem. If $\mu$ is a Gibbs state for $\Phi$ with inverse temperature $\beta$, and if $c>[2(v+1) / \beta m]^{1 / 2}$, then $\mu \mathfrak{X}(\tau)=1$, where

$$
\mathfrak{X}(\tau)=\left\{x \in \hat{\mathfrak{X}}: \exists r_{1}(x, \tau), r>r_{1} \Rightarrow \sup _{0 \leqq t \leqq \tau} V_{\max }\left(T_{r}^{t} x ; \Lambda(r)\right)<c(\log r)^{1 / 2}\right\} .
$$

Proof. Choose $\gamma<m \beta / 2$ positive so that $c^{2}>(v+1) / \gamma$. Then apply A.2 and the Borel-Cantelli lemma: with $\lambda=c(\log r)^{1 / 2}$ in A.2 one has

$$
\mu E_{r}(\tau) \leqq \operatorname{const}(1+\tau) r^{v} \exp \left[-\gamma c^{2} \log r\right] \leqq \operatorname{const}(1+\tau) r^{\nu-\gamma c^{2}} .
$$

A.4. Now for the time-evolution of the infinite system we establish bounds on the growth of $\sup _{0 \leqq t \leqq \tau} V_{\max }\left(T^{t} x ; \Lambda(s)\right)$ as $s$ tends to infinity. Let $x$ belong to both $\hat{\mathfrak{X}}$ (of 4.7) and $\mathfrak{X}(\tau)$ (of A.3), and take $s$ to be larger than $r_{0}(x, \tau)$ from 4.7 and $r_{1}(x, \tau)$ in A.3. Now let

$$
r(s)=\inf \left\{r: s+c\left(1+t_{0}^{2}\right)^{1 / 2}(\log r)^{\alpha} \leqq r / 4\right\} .
$$

The proof of 5.2 then shows that $T^{t} x \uparrow_{\Lambda(s)}=T_{r(s)}^{t} x \uparrow_{A(s)}$ if $0 \leqq t \leqq \tau$, and we have the simple estimate

$$
\sup _{0 \leqq t \leqq \tau} V_{\max }\left(T^{t} x ; \Lambda(s)\right) \leqq \sup _{0 \leqq t \leqq \tau} V_{\max }\left(T_{r}^{t} x ; \Lambda(r)\right)<c(\log r)^{1 / 2}
$$


The standard asymptotic development from (A.4.1) shows that there is a constant $b>0$ so that

$$
r(s) \leqq 4 s+b(\log s)^{\alpha},
$$

hence

$$
\log r(s) \leqq \log s+o(\log s)
$$

and this proves the following theorem.

A.5. Theorem. Let $\tau, \beta$ be positive, and let $c>(2(v+1) / m \beta)^{1 / 2}$. If $\mu$ is a Gibbs state for $\Phi$ with inverse temperature $\beta$, then for $\mu$-a.e. $x \in \mathfrak{X}$ there is an $s_{0}=s_{0}(x, \tau)$ such that if $s>s_{0}$ the following inequality holds:

$$
\sup _{0 \leqq t \leqq \tau} V_{\max }\left(T^{t} x ; \Lambda(s)\right)<c(\log s)^{1 / 2} .
$$

A.6. Remark. It can be shown that there is a subset of $\mathfrak{X}$ of full measure in every Gibbs state simultaneously, consisting of points $x$ for which (A.5.1) holds for $s$ sufficiently large depending on $x$, provided that the exponent $1 / 2$ is replaced by $1 / 2+\varepsilon$, for any $\varepsilon$ positive. In this case the positive constant $c$ is arbitrary. The proof is the obvious modification of A.3 and A.5, and we omit it.

\section{References}

1. Alexander,R.: The infinite hard-sphere system. Thesis, University of California, Berkeley, 1975

2. Bourbaki, N.: General topology, Part 2. Paris: Hermann 1966

3. Gowrisankaran, N.K.: Measurability of functions in product spaces. Proc. Amer. Math. Soc. 31, $485-488(1972)$

4. Lanford, O. E.III: One-dimensional systems of infinitely many Particles I: An existence theorem. Commun. math. Phys. 9, 169-181 (1968)

5. Lanford, O.E., III: One-dimensional systems of infinitely many particles II: Kinetic Theory. Commun. math. Phys. 11, 257-292 (1969)

6. Lanford, O.E., III: Time evolution of large classical systems. In: Dynamical systems, theory and applications. Lecture Notes in Physics, Vol. 38, (ed. J. Moser). Berlin-Heidelberg-New York: Springer 1975

7. Marchioro, C., Pellegrinotti,A., Presutti, E.: Existence of time evolution for $v$-dimensional statistical mechanics. Commun. math. Phys. 40, 175-186 (1975)

8. Ruelle, D.: Statistical mechanics. New York: Benjamin 1969

9. Schwartz, L.: Radon measures on arbitrary topological spaces and cylindrical measures. Oxford University Press, Tata Institute for Fundamental Research, 1973

10. Sinai, Ya.G.: Construction of the dynamics for one-dimensional systems of statistical mechanics. Teoret. Mat. Fiz. 11, 248 -258 (1972). English translation: Theoret. Math. Phys. 487 -494 (1973)

11. Sinai, Ya.G.: The construction of cluster dynamics for dynamical systems of statistical mechanics. Vestn. Mosk. Univ. Ser. I: Mat.-Meh. 29, 152-159 (1974). English translation: Mosc. Univ. Math. Bull. 29, 124-130 (1974)

Communicated by J. L. Lebowitz 